\title{
Análise dos critérios normativos de saúde e segurança do trabalho na manutenção de indústrias de celulose e papel
}

Denise Ransolin Soranso denise_soranso@hotmail.com Universidade Federal de Itajubá (UNIFEI), Itajubá, MG, Brasil.

Aryadne Miranda Gomes de Assis aryadne.assis@ufv.br Universidade Federal de Viçosa (UFV) Viçosa, MG, Brasil.

\section{Luciano José Minette} minette@ufv.br

Universidade Federal de Viçosa (UFV), Viçosa, MG, Brasil.

Julio César Costa Campos julio.campos@ufv.br Viçosa, MG, Brasil.

\begin{abstract}
A qualidade dos processos em indústrias de celulose e papel, e consequentemente, a qualidade do produto estão associadas ao desempenho dos serviços e dos trabalhadores durante a execução das atividades em seus postos de trabalho, seja ele administrativo, através tarefas burocráticas, de logística e de controle do fluxo de matéria-prima, ou atividades técnicas no ambiente fabril, como manutenção das máquinas, transporte de insumos e processamentos químicos. Dessa maneira, a busca por um ambiente seguro passa a fazer parte da estratégia empresarial, devido a influência social e econômica, sendo assim, a análise das condições de trabalho as quais os trabalhadores estão expostos é extremamente relevante para o sucesso da empresa. Neste contexto, o presente trabalho teve como objetivo do trabalho analisar as condições de trabalho das funções ligadas ao setor de manutenção de uma indústria de celulose e papel e verificar o atendimento das conformidades relacionadas a normatização de saúde e segurança do trabalho. Para isso, foi feita uma análise quantitativa dos dados, do setor de manutenção de uma indústria de celulose e papel, contidos no documento fornecido pelo Laboratório de Ergonomia do Departamento de Engenharia de Produção e Mecânica da Universidade Federal de Viçosa. Foram avaliados fatores os fatores ambientais (ruído, calor e iluminação) e as condições ergonômicas. Os resultados da análise das conformidades e não conformidades das funções revelou uma condição crítica no ambiente de trabalho relacionado ao setor de manutenção, indicando a necessidade de adoção de medidas corretivas.
\end{abstract}




\section{INTRODUÇÃO}

O setor florestal é mundialmente conhecido pelo fornecimento de energia e matéria-prima para diversas indústrias. E neste cenário, o Brasil se destaca por possuir recursos florestais abundantes e a segunda maior cobertura florestal do mundo, atrás apenas da Rússia, de acordo com o Sistema Nacional de Informações Florestais. Além disso, o país desenvolveu uma estrutura produtiva complexa no setor florestal, incluindo relações com produtores de equipamentos, insumos, projetos de engenharia e empresas de produtos florestais (SERVIÇO FLORESTAL BRASILEIRO, 2018).

Nos anos 70, com os incentivos fiscais para o reflorestamento, as florestas plantadas expandiram-se, contribuindo para o reaproveitamento de terras degradadas pela agricultura, sequestro de carbono, proteção do solo e da água e desenvolvimento de maquinário mais adequado. De acordo com o relatório anual da Indústria Brasileira de Árvores (IBÁ, 2017), a produção de celulose em 2016, atingiu 12,9 milhões de toneladas, representando 11,4 bilhões em tributos federais, estaduais e municipais e geração de empregos no país.

Com a expansão e consolidação da indústria de celulose no mercado mundial, toda a cadeia produtiva é beneficiada. Desde a plantação e extração da matéria, até o beneficiamento e entrega dos produtos aos clientes. Mas para que haja qualidade em todo o processo é necessário um acompanhamento e estudo intenso das etapas de trabalho, buscando a otimização dos processos e redução dos impactos ambientais e sociais.

Para isso, as empresas realizam estudos ergonômicos, que são apresentados na forma de um laudo, ou também uma Análise Ergonômica do Trabalho (AET), e avaliação dos riscos ambientais, apresentado como o Programa de Prevenção de Riscos Ambientais (PPRA). Esses estudos são extremamente importantes pois a qualidade das atividades realizadas durante a cadeia produtiva interfere diretamente na qualidade do produto entregue, tanto tratando-se da questão financeira quanto de questões sociais que envolvem saúde e segurança dos trabalhadores (BRASIL, 2019a).

Os riscos estão em toda a cadeia produtiva, e a exposição dos trabalhadores a eles é inevitável. No entanto, com o estudo dos mesmos através do levantamento quantitativo e qualitativo em cada processo, a indústria pode encontrar maneiras de minimiza-los garantindo a segurança do trabalhador durante a realização do seu trabalho. Essas ações são amparadas pelas Normas Regulamentadoras (NR) do Ministério do Trabalho, com destaque para as NR $15 \mathrm{e}$ 17 (BRASIL, 2019b, BRASIL, 2019c).

Devido à importância da manutenção de boas condições de trabalho ao longo dos processos produtivos das indústrias de celulose, esse trabalho tem como finalidade contribuir para conscientização sobre a existência de riscos nesse setor, a fim de diminuir as ocorrências de acidentes e doenças relacionadas ao trabalho, garantindo a otimização do processo produtivo e melhoria da qualidade final dos serviços e produtos. Nesse contexto, o objetivo do trabalho foi analisar as condições de trabalho das funções ligadas ao setor de manutenção de uma indústria de celulose e papel e verificar o atendimento das conformidades relacionadas a normatização de saúde e segurança do trabalho. 


\section{ÁREA DE ESTUDO E OBTENÇÃO DE DADOS}

O presente estudo foi desenvolvido por meio de um banco de dados fornecido pelo Laboratório de Ergonomia do Departamento de Engenharia de Produção e Mecânica da Universidade Federal de Viçosa (UFV). Os dados obtidos são referentes a avaliação realizada em uma empresa que tem como atividade principal a fabricação de celulose para a fabricação de papel. As atividades executadas na empresa variam entre funções administrativas, de engenharia, técnicas e de vigilância, totalizando 697 funcionários.

Para a avaliação das conformidades e não conformidades, foi feita uma filtragem do banco de dados das seguintes variáveis: ruído, calor, iluminação e condições ergonômicas. Na sequência, foi realizado o reconhecimento dessas variáveis por atividades da empresa. Devido ao grande número de funções dispostas no banco de dados fornecido para a realização deste trabalho, optou-se por realizar a análise das conformidades e não conformidades nos setores relacionados a atividade de manutenção, visto que, ao realizar um estudo prévio dos dados, foi verificado que essa atividade proporciona a ocorrência de um número considerável de riscos aos trabalhadores.

$\mathrm{Na}$ análise dos dados, verificou-se que dos 207 setores existentes na empresa, 37 estão diretamente ligados a atividades de manutenção. E, das 319 funções avaliadas, 99 relacionam-se a manutenção. Desse modo, cerca de $31 \%$ das funções exercidas na empresa encontram-se ligadas a manutenção. Com base nessas informações, foi realizado o levantamento e identificação de todos os setores e funções ligadas a realização de manutenção na empresa, conforme listado no Anexo 1.

\section{CRITÉRIOS E TÉCNICAS DE AVALIAÇÃC}

Para avaliar as conformidades e não conformidades de atendimento dos critérios normativos de saúde e segurança do trabalho no setor de manutenção, foram utilizadas referências normativas como: as Normas Regulamentadoras (NR), Normas da Associação Brasileira de Normas Técnicas (NBR) e Normas de Higiene Ocupacional (NHO). A avaliação associou a variável estudada com o critério normativo empregado para avaliação do risco no ambiente de trabalho. Os dados analisados foram referentes ao ruído (conforto acústico), calor, iluminação e condições biomecânicas.

\section{RUÍDO}

A exposição ao ruído foi avaliada conforme a NR 17 (Ergonomia) e NBR 10152 (Níveis de ruído para conforto acústico). Segundo as normas, para as atividades consideradas o nível de ruído aceitável para efeito de conforto acústico deve ser de até $65 \mathrm{~dB}$. 
A partir da definição do critério normativo, cada função foi avaliada individualmente e foi verificado se o valor encontrado estava acima do valor estabelecido pela norma. Para os

valores acima do estabelecido pela norma, a função foi considerada em não conformidade. E para as funções com valores inferiores ou iguais ao estabelecido pela norma, foram consideradas conformes.

\section{CALOR}

Para análise do calor foi considerado o valor de Índice de Bulbo Úmido Termômetro de Globo (IBUTG), conforme normatizado na NR 15 (Atividades e Operações Insalubres). A norma estabelece que para caracterizar a exposição do trabalhador ao calor em ambientes internos ou externos, deve-se utilizar este índice. Neste caso, para verificar as conformidades e não conformidades referentes a exposição ao calor, foi considerado o limite de tolerância, em função do regime de trabalho, sendo verificado diferentes valores de limites para as funções avaliadas.

Nas situações em que as funções proporcionaram valores de IBUTG acima do limite normatizado, esta foi classificada como não conforme. E nos casos em que os a valores encontravam-se abaixo do limite da norma, foi feita a classificação como em conformidade:

\section{ILUMINAÇÃO}

A conformidade da variável iluminação foi avaliada, conforme critérios da NBR ISO 8995-1. A norma estabelece que a iluminância no entorno imediato deve estar relacionada com a iluminância da área de tarefa, e convém que proveja uma distribuição bem balanceada da iluminância no campo de visão. A iluminância mantida das áreas do entorno imediato pode ser mais baixa que a iluminância da área da tarefa, mas não pode ser inferior aos valores contidos na Quadro 1.

Quadro 1 - Valores de iluminância do local de trabalho

\begin{tabular}{|c|c|}
\hline Iluminância da tarefa (lux) & Iluminância do entorno imediato (lux) \\
\hline$\geq 750$ & 500 \\
\hline 500 & 300 \\
\hline 300 & 200 \\
\hline$\leq 200$ & Mesma iluminância da área da tarefa. \\
\hline
\end{tabular}

Fonte: NBR 8995-1 (2013)

\section{CONDIÇÕES BIOMECÂNICAS DO POSTO DE TRABALHO}

Para analisar as conformidades e não conformidades relacionadas as condições biomecânicas do posto de trabalho, foi utilizado os critérios de 
avaliação, obtidos por meio da aplicação do checklist de Avaliação Simplificada. A metodologia de avaliação sugere a classificação da condição biomecânica, em função da pontuação obtida ao aplicar o método. Nesse sentido, as funções avaliadas que proporcionaram resultados (pontos) acima de 5 , foram consideradas em conformidade, pois o método de avaliação aplicado, as considerou como condições ergonômicas de razoável a excelente. E as que apresentaram resultado abaixo de 5 , foram julgadas em não conformidades, pois a condição ergonômicas, nestes casos, foi de ruim a péssima, conforme critérios do Quadro 2.

Quadro 2 - Critérios de interpretação das condições ergonômicas de trabalho

\begin{tabular}{|l|l|c|}
\hline \multicolumn{1}{|c|}{ Pontuação } & \multicolumn{1}{|c|}{ Classificação } & \multirow{2}{*}{ Julgamento } \\
\hline 11 a 13 Pontos & Condições Ergonômicas Excelentes & \multirow{2}{*}{ Em conformidade } \\
\hline 8 a 10 Pontos & Boa Condição Ergonômica & \multirow{2}{*}{ Não conformidade } \\
\hline 6 a 7 Pontos & Condição Ergonômica Razoável & \\
\hline 4 a 5 Pontos & Condição Ergonômica Ruim & \\
\hline Menos de 4 Pontos & Condição Ergonômica Péssima & \\
\hline
\end{tabular}

Fonte: Couto (2013)

\section{RESULTADOS E DISCUSSÃO}

A partir dos fatores analisados, foi feito um estudo quantitativo e verificado, para cada um dos critérios normativos/metodológico, se a situação da função encontrava-se em conformidade ou não conformidade. Os resultados das variáveis consideradas para análise de atendimento dos critérios normativos estão na Figura 1.

Figura 1 - Resultado da avaliação quantitativa das conformidades e não conformidades dos fatores ambientais e das condições ergonômicas no local de trabalho

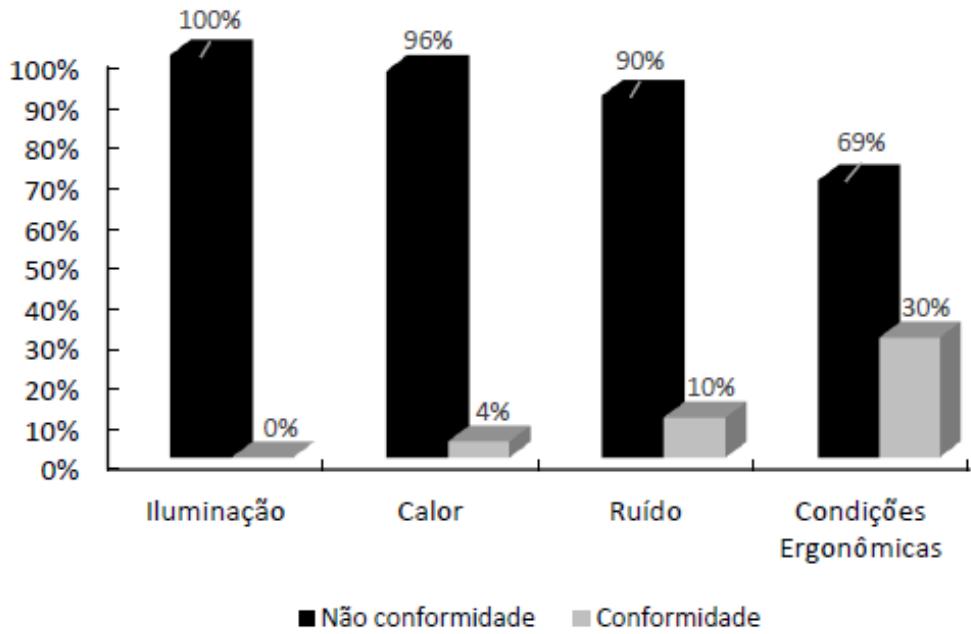

Fonte: Autoria Própria (2019) 
De acordo com os resultados, é possível observar que a maioria das condições das quais os trabalhadores estão expostos não estão em conformidade com os critérios normativos/metodológico considerados. Das 72 funções avaliadas no quesito iluminação, $100 \%$ encontram-se em não conformidade; das 60 funções que proporcionaram exposição ao calor, 96,6 \% estão em não conformidade e das 99 funções com exposição ao ruído, $90 \%$ encontra-se em não conformidade.

O alto nível de não conformidades dos fatores ambientais analisados (iluminação, calor e ruído), torna a situação da empresa preocupante, pois além de não oferecer aos trabalhadores condições adequadas de trabalho, acometendo os mesmos as situações de danosas a sua saúde. A empresa, pode ainda, ser penalizada pelos órgãos fiscalizadores, pelo não atendimento de critérios normativos de saúde e segurança do trabalho, tendo esta que pagar multas e indenizações.

$\mathrm{Na}$ análise do ruído, a maior preocupação é o risco potencial de surdez ocupacional (FUNDACENTRO, 2019). O ruído age diretamente sobre o sistema nervoso do trabalhador, podendo causar: fadiga nervosa, hipertensão, modificação do ritmo cardíaco, perda temporária ou definitiva da audição. Nesses casos, recomenda-se a adoção de medidas de proteção, como o enclausuramento da máquina geradora de ruído, utilização de Equipamentos de Proteção Individual (EPI); realização de exames audiômetros periódicos, para acompanhamento da saúde do trabalhador, e revezamento das atividades de trabalho próximas as fontes de ruído, com intuito de reduzir o tempo de exposição (KROEMER; GRANDJEAN, 2005; SILVEIRA et al., 2007; FUNDAÇÃO OSWALDO CRUZ, 2019).

Em tratando-se do calor, o alto percentual de não atendimento das conformidades estabelecidos pelo critério normativo, também podem refletir em danos à saúde do trabalhador. A desidratação, as convulsões, o enjoo, as dores de cabeça, as tonturas e cãibras, são alguns exemplos de danos ao qual o trabalhador pode estar submetido em função da exposição a este agente. A adoção de medidas, por vezes simples, mas que podem minimizar o estresse térmico, como: executar as tarefas mais pesadas na parte da manhã, período em que a radiação solar é mais amena; utilização de vestimentas, confeccionadas com tecidos mais leves, permitindo a circulação de ar no corpo e instalação de equipamentos de ventilação (FLORES,2017).

No que tange a questão da iluminação, a NR 17 estabelece que em todos os locais de trabalho deve haver iluminação adequada, natural ou artificial, geral ou suplementar, apropriada à natureza da atividade, (BRASIL, 2019c). Além disso, as não conformidades dos requisitos previstos pela NBR 8995-1 (Iluminação de Ambiente de Trabalho), podem provocar desde problemas físicos até emocionais, como: irritação nos olhos e cansaço visual e distúrbios emocionais.

Em relação as condições ergonômicas, das 99 funções avaliadas, 69 apresentaram conformidades e 30 foram julgadas como em não conformidade. Apesar, da maioria das funções proporcionar um condição ergonômica satisfatória, o número de funções com condições ruins ou péssima é elevado, demostrando a necessidade de verificação e adequar as condições de trabalho. Nesse sentido, destaca-se que a importância de identificação e aplicação de estratégias de ação, visando a melhoria das condições de trabalho para a 
prevenção do surgimento de doenças e, consequentemente, afastamento de trabalhadores.

Os efeitos a longo prazo de condições ergonômicas inadequadas são abundantes, dentre os quais destaca-se os problemas nas articulações, que comprometem principalmente a coluna vertebral (COUTO, 1996). Contudo, tal condição não afeta somente a saúde do próprio trabalhador, mas também gera graves consequências sociais, como o absenteísmo, a mudança de profissão por incapacidade laboral e gastos previdenciários (MERINO, 1996).

Nesse contexto, sugere-se a implementação de ações na empresa visando a prevenção e minimizar das condições de riscos aos trabalhadores. Práticas, como a realização de ginástica laboral e treinamentos, acompanhamento da saúde dos trabalhadores e estabelecimento de sistemas de pausas para descanso, são alguns dos exemplos que podem ser aplicados em ambientes de trabalho, visando a sua melhoria.

\section{CONCLUSÕES}

A análise das conformidades e não conformidades das funções revelou uma condição crítica no ambiente de trabalho relacionado ao setor de manutenção, haja vista, que o número de não conformidades foi alto, chegando ser total em algumas variáveis avaliadas, o que indica a necessidade de adoção de medidas corretivas a fim de regularizar e melhorar as condições de trabalho.

O grande número de não conformidade, além de gerar um problema para a própria empresa do ponto de vista financeiro (possíveis multas devido ao não cumprimento da legislação), gera como consequência um problema social decorrente dos riscos que os trabalhadores estão expostos, a prováveis acidentes, lesões e afastamentos. 


\title{
Analysis of normative criteria for health and safety at work in the maintenance of the cellulose and paper industries
}

\begin{abstract}
The quality of processes in the pulp and paper industries and, consequently, product quality are associated with the performance of services and workers during the execution of activities at their jobs, whether administrative, through bureaucratic, logistic and logistics tasks. control of the flow of raw materials, or with technical activities in the manufacturing environment, such as machine maintenance, transportation of inputs and chemical processing. Thus, the search for a safe environment becomes part of the business strategy, due to social and economic influence, thus, the analysis of the working conditions to which workers are exposed is extremely relevant to the success of the company. In this context, the present work aimed to analyze the working conditions of the functions linked to the maintenance sector of a pulp and paper industry and to verify compliance with the standards related to occupational health and safety. For this, a quantitative analysis of the data from the maintenance sector of a pulp and paper industry was made, contained in the document provided by the Ergonomics Laboratory of the Department of Production Engineering and Mechanics of the Federal University of Viçosa. Factors were evaluated environmental factors (noise, heat and lighting) and ergonomic conditions. The results of the conformity and nonconformities analysis of the functions revealed a critical condition in the work environment related to the maintenance sector, indicating the need for corrective measures.
\end{abstract}

Nonconformities. Environmental factors. Ergonomic conditions. 


\section{REFERÊNCIAS}

ASSOCIAÇÃO BRASILEIRA DE NORMAS TÉCNICAS - NBR 8995-1: Iluminação de Ambiente de Trabalho, Rio de Janeiro, 2013.

ASSOCIAÇÃO BRASILEIRA DE NORMAS TÉCNICAS - NBR 10152: Níveis de ruído para conforto acústico, Rio de Janeiro, 2017.

BRASIL. MINISTÉRIO DO TRABALHO E EMPREGO. Normas Regulamentadoras de Segurança e Medicina do Trabalho. NR 09: Programa de Prevenção de riscos Ambientais. Disponível em:

https://enit.trabalho.gov.br/portal/images/Arquivos_SST/SST_NR/NR-09.pdf. Acesso em: 15 de junho de 2019a.

BRASIL. MINISTÉRIO DO TRABALHO E EMPREGO. Normas Regulamentadoras de Segurança e Medicina do Trabalho. NR 15 - Atividades e operações insalubres. Disponível em:

https://enit.trabalho.gov.br/portal/images/Arquivos_SST/SST_NR/NR-15.pdf. Acesso em: 15 de junho de 2019b.

BRASIL. MINISTÉRIO DO TRABALHO E EMPREGO. Normas Regulamentadoras de Segurança e Medicina do Trabalho. NR 17 - Ergonomia Disponível em: https://enit.trabalho.gov.br/portal/images/Arquivos_SST/SST_NR/NR-17.pdf. Acesso em: 15 de junho de 2019c.

COUTO, H. A. Ergonomia aplicada ao trabalho: o manual técnico da máquina

humana. Belo Horizonte: Ergo, v. 2. 1996. 383 p.

FLORES, C. Exposição Ocupacional ao Calor e Estresse Térmico. Artigo publicado em Saber SST - Difusão de conhecimento em Saúde, Segurança e Higiene Ocupacional, publicado no dia 25 de janeiro de 2017. Disponível em https://www.saudeesegurancanotrabalho.org/exposicao_calor/. Acesso em: 20 de junho de 2019.

FUNDAÇÃO OSWALDO CRUZ. Riscos Físicos. Disponível em http://www.fiocruz.br/biosseguranca/Bis/lab_virtual/riscos_fisicos.html. Acesso em: 20 de junho de 2019.

IBÁ. INSTITUTO BRASILEIRO DE ÁRVORES - Relatório Anual de 2017. Disponível em http://iba.org/pt/. Acesso em :15 de abril de 2018. 
KROEMER, K. H. E; GRANDJEAN, E. Manual de ergonomia: Adaptando o trabalho ao homem. 5.ed. Porto Alegre: Bookman, 2005, 327 p.

MERINO, E. A. D. Efeitos agudos e crônicos causados pelo manuseio e movimentação de cargas no trabalhador. 1996. 128 f. Dissertação (Mestrado em Engenharia de Produção e Sistemas) - Universidade Federal de Santa Catarina, Florianópolis, 1996.

SILVEIRA, J. C. M. da; FERNANDES, H. C.; RINALDI, P. C. N.; MODOLO, A. J. Níveis de ruído em função do reio de afastamento emitido por diferentes equipamentos em uma oficina agrícola. Engenharia na Agricultura, Viçosa, v. 15, n.1, p.66-74, jan./mar. 2007.

SERVIÇO FLORESTAL BRASILEIRO. Florestas Plantadas. Disponível em http://www.florestal.gov.br/snif/entenda-o-snif/o-portal. Acesso em: 15 de Abril de 2018. 


\section{ANEXO}

Anexo 1 - Setores e funções avaliadas relacionadas a atividade de manutenção de uma empresa de celulose e papel

\begin{tabular}{|c|c|c|}
\hline Setor & & Função \\
\hline \multirow{3}{*}{ Manutenção Mecânica das Áreas } & 1 & Mecânico de Manutenção I \\
\hline & 2 & Mecânico de Manutenção II \\
\hline & 3 & Mecânico de Manutenção IV \\
\hline Manutenção Mecânica de oficina & 4 & Auxiliar almoxarifado \\
\hline Gerência de Manutenção Industrial & 5 & Desenhista Industrial \\
\hline Carga e Transp. Rodoviário - Biomassa & 6 & Mecânico de Manutenção de motosserra \\
\hline \multirow{2}{*}{$\begin{array}{l}\text { Manutenção Via Perm / Equipamentos } \\
\text { Ferroviários }\end{array}$} & 7 & Mecânico de Equipamentos móveis \\
\hline & 8 & Mecânico de Locomotiva \\
\hline $\begin{array}{l}\text { Manutenção Via Perm / Equipamentos } \\
\text { Ferroviários }\end{array}$ & 9 & Eletricista Locomotiva \\
\hline Regência de Manutenção Industrial & 10 & Planejador de Manutenção I \\
\hline \multirow{4}{*}{$\begin{array}{l}\text { Coordenação de Manutenção Elétrica e } \\
\text { Instrumentação }\end{array}$} & 11 & Supervisor de Manutenção Elétrica \\
\hline & 12 & Engenheiro de Manutenção I \\
\hline & 13 & Técnico eletricista III \\
\hline & 14 & Técnico Esp. em Instrumentação \\
\hline \multirow{2}{*}{ Oficina de Apoio } & 15 & Auxiliar de Planejamento e Manutenção \\
\hline & 16 & Supervisor de Manutenção Mecânica \\
\hline \multirow{8}{*}{ Gerencia de Manutenção Industrial } & 17 & Gerente de Manutenção \\
\hline & 18 & Coordenador de Engenharia De Manutenção \\
\hline & 19 & Planejador de Manutenção II \\
\hline & 20 & Engenheiro de Manutenção I \\
\hline & 21 & Engenheiro de Manutenção II \\
\hline & 22 & Auxiliar de Manutenção Mecânica \\
\hline & 23 & Auxiliar de Manutenção Elétrica \\
\hline & 24 & Técnico de Manutenção Mecânica I \\
\hline \multirow{2}{*}{ Manutenção Mecânica das Áreas } & 25 & Assistente técnico Administrativo \\
\hline & 26 & Coordenador de Manutenção Mecânica \\
\hline
\end{tabular}

\begin{tabular}{|c|c|}
\hline \multirow{2}{*}{ Gerência de Manutenção Industrial } & 27 Inspetor de Manutenção de Equipamentos I \\
\hline & 28 Supervisor de Inspeção Mecânica Industrial \\
\hline \multirow{4}{*}{ Manutenção Mecânica das Áreas } & 29 Inspetor de Manutenção de Equipamentos I \\
\hline & 30 Inspetor de Manutenção de Equipamentos II \\
\hline & 31 Técnico Especializado \\
\hline & 32Técnico II \\
\hline \multirow{2}{*}{ Gerência de Manutenção Industrial } & 33Técnico Especializado \\
\hline & 34 Técnico II \\
\hline Oficina de apoio & 35 Técnico de Manutenção mecânica II \\
\hline \multirow[t]{2}{*}{ Oficina de apoio - Prédio Oficina Central } & 36 Supervisor de Içamento de Apoio de Manutenção \\
\hline & 37 Consulto de Manutenção Mecânica \\
\hline Oficina de apoio - Ferramenta & 38 Mecânico de Manutenção II \\
\hline \multirow{2}{*}{ Oficina de apoio } & 39 Torneiro Mecânico de Manutenção II \\
\hline & 40 Torneiro Mecânico de Manutenção III \\
\hline \multirow{2}{*}{ Oficina de apoio } & 41 Soldador de Manutenção II \\
\hline & 42 Soldador de Manutenção III \\
\hline Manutenção Mecânica das Áreas & 43 Lubrificador \\
\hline \multirow{3}{*}{ Oficina de Apoio } & 44 Mecânico de Manutenção I \\
\hline & 45 Mecânico de Manutenção II \\
\hline & 46 Técnico de Manutenção Mecânica II \\
\hline \multirow{2}{*}{ Manutenção Mecânica das Áreas } & 47 Caldeireiro II \\
\hline & 48 Caldeireiro III \\
\hline \multirow{2}{*}{$\begin{array}{l}\text { Coordenador de Manutenção Elétrica e de } \\
\text { Instrumentação }\end{array}$} & 49 Mecânico de Refrigeração I \\
\hline & 50 Mecânico de Refrigeração II \\
\hline Manutenção Mecânica das Áreas & 51 Mecânico de Manutenção I \\
\hline \multirow{4}{*}{ Manutenção Mecânica das Áreas } & 52 Auxiliar de Manutenção Mecânica \\
\hline & 53 Mecânico de Manutenção II \\
\hline & 54 Mecânico de Manutenção III \\
\hline & 55 Mecânico de Manutenção IV \\
\hline Manutenção Mecânica das Áreas & 56 Caldeireiro II \\
\hline \multirow{2}{*}{ Gerência de Manutenção Industrial } & 57 Planejador de Manutenção I \\
\hline & 58 Supervisor de Manutenção Mecânica \\
\hline Oficina de Apoio & 59 Mecânico de Manutenção II \\
\hline
\end{tabular}




\begin{tabular}{|c|c|}
\hline \multirow{3}{*}{ Manutenção Mecânica das Áreas. } & 60 Mecânico de Manutenção I \\
\hline & 61 Mecânico de Manutenção II \\
\hline & 62 Mecânico de Manutenção IV \\
\hline Manutenção Mecânica das Áreas & 63 Soldador de Manutenção III \\
\hline \multirow{2}{*}{ Manutenção Mecânica das Áreas } & 64 Caldeireiro I \\
\hline & 65 Caldeireiro II \\
\hline \multirow{4}{*}{ Manutenção Mecânica das Áreas } & 66 Auxiliar de Manutenção Mecânica \\
\hline & 67 Mecânico de Manutenção I \\
\hline & 68 Mecânico de Manutenção II \\
\hline & 69 Mecânico de Manutenção III. \\
\hline \multirow{2}{*}{ Manutenção Mecânica das Áreas } & 70 Soldador de Manutenção II \\
\hline & 71 Soldador de Manutenção III. \\
\hline Manutenção Mecânica das Áreas & 72 Supervisor de Manutenção Mecânica \\
\hline \multirow{3}{*}{ Oficina de Apoio } & 73 Mecânico de Manutenção I \\
\hline & 74 Mecânico de Manutenção II \\
\hline & 75 Mecânico de Manutenção III \\
\hline Manutenção Mecânica das Áreas & 76 Mecânico de Manutenção IV \\
\hline Manutenção Mecânica das Áreas & 77 Caldeireiro II \\
\hline \multirow{2}{*}{ Oficina de Manutenção } & 78 Técnico em Manutenção Mecânica \\
\hline & 79 Auxiliar de Manutenção Mecânica \\
\hline \multirow{2}{*}{ Manutenção Mecânica das Áreas } & 80 Mecânico de Manutenção I \\
\hline & 81 Mecânico de Manutenção IV \\
\hline Manutenção Mecânica das Áreas & 82 Soldador de Manutenção II \\
\hline \multirow{4}{*}{$\begin{array}{l}\text { Coordenador de Manutenção Elétrica e } \\
\text { Instrumentação }\end{array}$} & 83 Auxiliar de Manutenção elétrica \\
\hline & 84 Eletricista de Manutenção I \\
\hline & 85 Eletricista de Manutenção II \\
\hline & 86 Eletricista de Manutenção III \\
\hline & 87 Técnico Eletricista I \\
\hline & 88 Técnico Eletricista II \\
\hline & 89 Técnico Eletricista III \\
\hline & 90 Técnico de Instrumentação I \\
\hline & 91 Técnico de Instrumentação II \\
\hline & 92 Técnico de Instrumentação III. \\
\hline $\begin{array}{l}\text { Coordenador de Manutenção Elétrica e } \\
\text { Instrumentação. }\end{array}$ & 93 Técnico Eletricista I \\
\hline \multirow{3}{*}{$\begin{array}{l}\text { Coordenador de Manutenção Elétrica e } \\
\text { Instrumentação. }\end{array}$} & 94 Técnico de Instrumentação I \\
\hline & 95 Técnico de Instrumentação II \\
\hline & 96 Técnico de Instrumentação III. \\
\hline $\begin{array}{l}\text { Manutenção de Via } \\
\text { Permanente/Equipamentos Ferroviários }\end{array}$ & 97 Soldador de Manutenção I \\
\hline Manutenção Mecânica das Áreas & 98 Caldeireiro II \\
\hline Manutenção Mecânica das Áreas & 99 Caldeireiro III \\
\hline
\end{tabular}

